\title{
Role of Early Intervention in Improving the Clinical Outcome of Acute Pancreatitis by Image Guided Percutaneous Catheter Drainage of Pancreatic Collections
}

\author{
Chittoor Ramya Sudha ${ }^{\odot 1}$, Yugandhar Samireddypalle ${ }^{\odot 2}$, Umamaheswara V Reddy ${ }^{\circledR 3}$, Sagar Dembla ${ }^{\circledR 4}$, Gurudutt P. Varty ${ }^{\circ}$, Ankita \\ Sanjeev Dhawan ${ }^{\oplus 5}$, Amitha K S Vikrama ${ }^{\oplus 6}$ \\ ${ }^{1}$ Post Graduate, Department of Radiology, Narayana Medical College, Nellore, Andhra Pradesh, India, ${ }^{2}$ Senior Resident, Department of Radiology, Narayana Medical \\ College, Nellore, Andhra Pradesh, India, ${ }^{3}$ Assistant Professor, Department of Radiology, Narayana Medical College, Nellore, Andhra Pradesh, India, ${ }^{4}$ Senior Resident, \\ Department of Medical Gastroenterology, Narayana Medical College, Nellore, Andhra Pradesh, India, ${ }^{5}$ Senior Resident, Department of Surgical Gastroenterology, \\ Narayana Medical College, Nellore, Andhra Pradesh, India, ${ }^{6} \mathrm{Head}$, Department of Radiology, Apollo Hospitals, Bangalore, Karnataka, India.
}

\section{Abstract}

Background: To assess the role of image guided percutaneous drainage of acute pancreatic collections in influencing the clinical outcome of patients. Subjects and Methods: This is a prospective study done in the department of intervention radiology, Narayana medical college. About 20 cases have been performed in our institution from January 2019 to December 2019. All these drainage procedures have been done under CT guidance. All these cases were strictly followed during their hospital course. Clinical success has been defined as control of sepsis and prevention of MODS without the requirement of surgical debridement. Results: Out of twenty cases, which we intervened, there was an improvement in clinical outcome in fourteen cases with the prevention of septic shock and multiorgan failure. Three cases were critically ill at the time of admission and succumbed to MODS (Multiple organ dysfunction syndrome). The other three cases required additional surgical intervention-debridement. In which, there was increased morbidity with delayed recovery in two cases, and one case developed MODS and expired. Conclusion: Image guided Percutaneous catheter drainage is a safe and effective technique to drain pancreatic collections. Adequate knowledge regarding the interpretation of imaging findings, time to intervene, adequate expertise for performing the procedure, close follow up with regular clinical assessment is vital in the management of patients in acute pancreatitis. Early and aggressive intervention helps reduce the morbidity and mortality by preventing the evolution of MODS.

Keywords: Pancreatitis, Multi-organ dysfunction Syndrome

Corresponding Author: Yugandhar Samireddypalle, Senior Resident, Department of Radiology, Narayana Medical College, Nellore, Andhra Pradesh, India.

E-mail: yugu.samireddypalle@gmail.com

Received: 11 April 2020

Revised: 16 May 2020

Accepted: 25 May 2020

Published: 05 July 2020

\section{Introduction}

Acute necrotizing pancreatitis corresponds to $10-15 \%$ of all cases of acute pancreatitis. ${ }^{[1]}$ It was frequently complicated by a systemic inflammatory response syndrome and multisystem organ failure and infections. Infectious pancreatic necrosis (IPN) is the most dreaded and severe complication causing $80 \%$ of the deaths associated with AP. ${ }^{[1-5]}$

The surgical debridement has been the mainstay of treatment of patients with acute necrotizing pancreatitis traditionally. However, such aggressive treatment is associated with significant complications and mortality in 11-39\% of patients. This high rate has been primarily attributed by the presence of comorbid medical conditions and multisystem organ failure which is derived from a systemic response to pancreatitis. ${ }^{[6-11]}$

Alternative nonsurgical techniques like percutaneous catheter drainage (PCD) have promising results of PCD in the treatment of the other complications which are routinely associated with AP, such as acute necrotising fluid collections. Limited studies have evaluated the role of CT-guided percutaneous catheter drainage in the care of patients with infected pancreatic necrosis. ${ }^{[12-15]}$ Compared with open surgery, PCD could effectively reduce the hospital stay and average hospital cost and avoid surgically related complications, such as wound infection and incisional hernia. ${ }^{[5-15]}$ 


\section{Subjects and Methods}

\section{Subjects}

The prospective study was performed from January 2019 to December 2019 after taking informed consent. There were 20 patients (all were men, the median age of 37 years) with a diagnosis of acute necrotising pancreatitis who underwent percutaneous catheter drainage via CT guidance and have been followed up. The cause of the pancreatitis was alcoholism in all cases at our center. Institutional ethical review board approval was obtained for this study.

Assessment of the patient's clinical condition at initial percutaneous CT guided PCD was dependent on the criteria based on the revised classification of Acute Pancreatitis 2012. The revised classification identifies early and late phases of the disease and classifies into mild, moderate, and severe acute pancreatitis. ${ }^{[4]}$ The mild acute pancreatitis has no organ failure, no local or systemic complications. The moderate acute pancreatitis has transient organ failure or local complications or exacerbation of comorbid diseases for less than 48 hours. The severe acute pancreatitis has persistent organ failure for more than 48 hours. ${ }^{[4]}$

In our center, we aimed at providing early and aggressive intervention for all patients with sizeable pancreatic collections, followed by regular upsizing of the catheter.

\section{Procedure}

Referral for radiological intervention has been based on clinical suspicion of infection raised by fever, leucocytosis and associated renal or cardiac compromise, or general clinical deterioration which is not responding to conservative medical care. The referral is also given if there is a sizeable pancreatic collection on imaging with rapid abdominal distension, irrespective of the clinical parameters.

The decision for PCD was based on guidelines according to the American College of Radiology appropriateness criteria with radiological imaging suggesting a liquefied drainable collection, collections larger than $5 \mathrm{~cm}$, rapidly enlarging collections, infected collections and collections causing obstruction. Infection in the collection was assessed on the imaging picture (the collection density has higher Hounsfield Units than water or the collection with gas) and in indeterminate cases with prior needle aspiration. ${ }^{[14,15]}$

\section{Drainage procedure}

PCD was performed under CT guidance, using local anaesthesia in strict sterile conditions. The Seldinger technique has been systematically used to place a catheter in the necrotic collections via the most direct transperitoneal route, without intervening bowels and solid organs. Retrogastric and lesser sac collections have been referred to the gastroenterologist for endoscopic interventions.

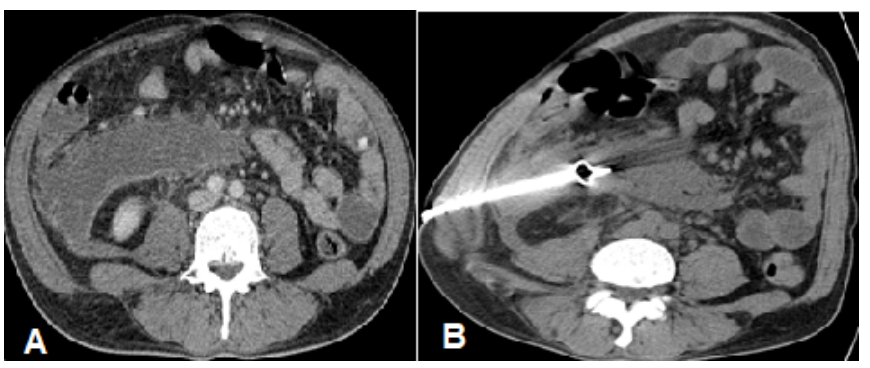

Figure 1: A 57-year-old man with moderately severe necrotizing pancreatitis. A. Axial contrast-enhanced CT image obtained 8 days after onset of symptoms showing acute necrotic fluid accumulation in right anteriorpararenal space extending into the right paracolic gutter (represented by the star symbol), B. Axial plain CT image obtained after insertion of malecot's catheter into the necrotic collection (depicted by white arrow)

To start with One or more 16- French malecot catheters were placed to aspirate the cavity fluid and then continuously irrigated with $1 \mathrm{~L} /$ drain/day of normal saline. Malecot catheters have been preferred due to less frequency of clogging. Later upsizing was done with the new larger bore catheter (Upto 28 French malecot), depending upon the amount drained and residual collection. Continuous and abundant daily irrigation was started, ranging from 0.5 to 1.5 L/drain/day of normal saline, depending on the size of the collection. If the drainage was inadequate, further upsizing of the catheter was performed up to 36 french ICD tube, under fluoro guidance, with the help of urethral dilators. The removal of the catheters and stoppage of the drainage was based on clinical improvement demonstrated by the control of the sepsis and hemodynamic stability, resolution of the necrotic cavity on CT scan, and the amount of drain fluid was less than 10 $\mathrm{mL} /$ day. The mean time between presentation with pancreatitis and the first interventional procedure was 7 days.

\section{Results}

Out of twenty cases, which we intervened, there was an improvement in clinical outcome in fourteen cases with the prevention of septic shock and multiorgan failure. Three cases were critically ill at the time of admission and succumbed to MODS (Multiple organ dysfunction syndrome). The other three cases required additional surgical interventiondebridement. In which, one case developed MODS and expired.

The mean hospital stay for our patients who were treated by PCD was 34.6 days (range 20 to 54 days), were followed up on OPD basis regularly. ICU hospitalisation has been required for 6 of the 20 patients out of which 4 succumbed to death. 


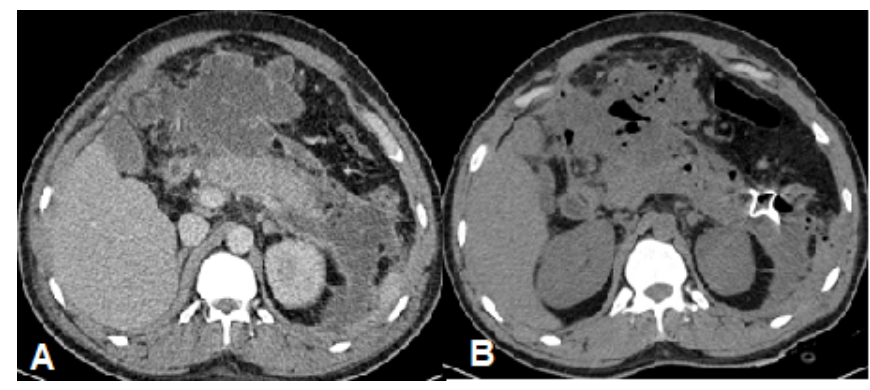

Figure 2: A 32-year-old man with moderately severe alcoholic necrotizing pancreatitis. A. Axial contrastenhanced CT image obtained 6 days after onset of symptoms showing acute peripancreatic fluid accumulation (represented by the star symbol) extending anteriorly into the greater sac, transverse mesocolon, and posteriorly into left anterior pararenal space and paracolic gutter with thickening of left pararenal facia (represented by the arrow symbol). Few air foci noted within the collection, suggesting infection. B. Axial plain CT image obtained after insertion of malecot's catheter into the necrotic collection (depicted by white arrow).
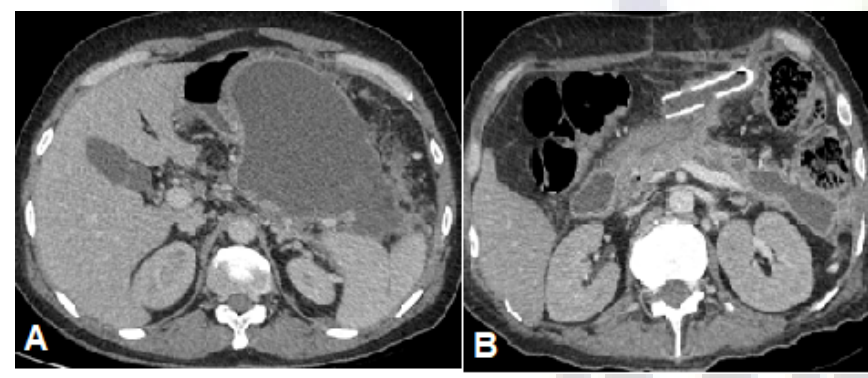

Figure 3: A 49-year-old man with the severe necrotizing pancreatitis. A. Axial contrast-enhanced CT image obtained 7 days after onset of symptoms showing acute peripancreatic fluid accumulation extending anteriorly into lesser sac, transverse mesocolon; superiorly into the lesser sac (represented by the star symbol) and posteriorly into left anterior pararenal space and paracolic gutter. B. Axial contrast-enhanced CT image obtained after insertion of ICD tube into the necrotic collection (depicted with the white arrow). ICD was placed by regular upsizing of the catheters.

The Ranson score was less than 3 for 9 patients, 3-5 for 8 patients and more than 6 for 3 patients. 14 patients fall under the moderately severe category and 6 patients in the severe category as per revised Atlanta classification of acute pancreatitis.

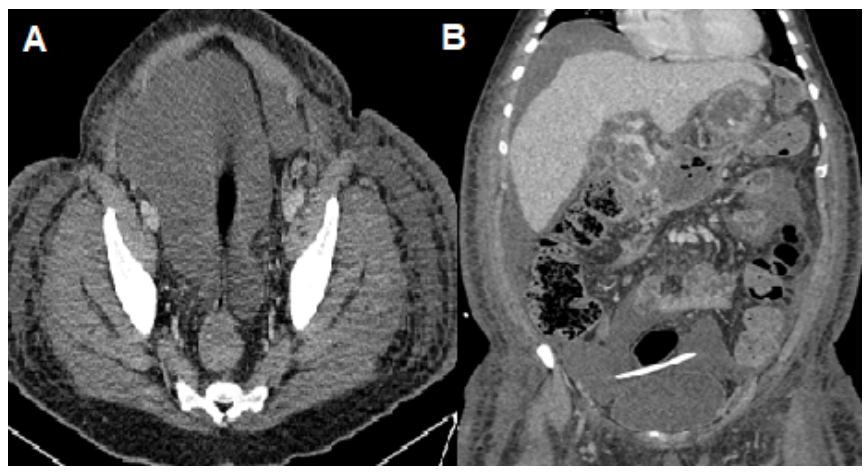

Figure 4: A 29-year-old man with the severe necrotizing pancreatitis. A. Axial contrast-enhanced CT image obtained 14 days after onset of symptoms showing fluid accumulation in the pelvis. (represented by the star symbol). B. Coronal contrast-enhanced CT image obtained after insertion of pigtail catheter into the pelvic fluid. (depicted by white arrow).

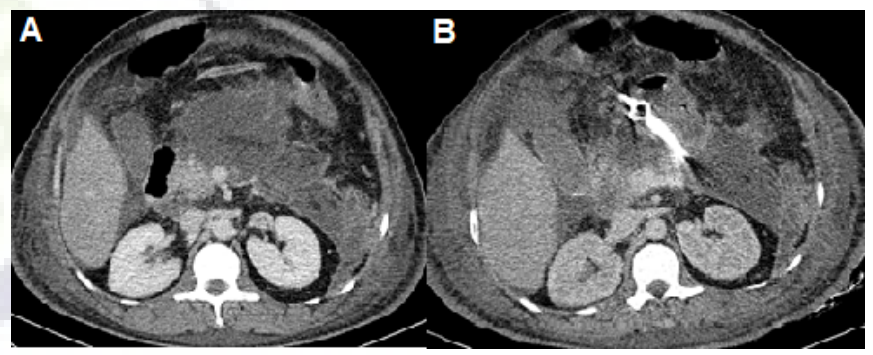

Figure 5: A 36-year-old man with severe necrotizing pancreatitis. A. Axial contrast-enhanced CT image obtained 9 days after onset of symptoms showing necrosis of pancreas and acute peripancreatic fluid accumulation (represented by the star symbol) extending into left anterior pararenal space and paracolic gutter. B. Axial contrastenhanced CT image obtained after insertion of Malecot's catheter into the necrotic collection (depicted by white arrow).

Among all 20 patients with acute necrotizing pancreatitis managed with primary CT-guided percutaneous catheter drainage, $14(70 \%)$ were treated successfully with drainage alone (Category A). These 14 patients referred for PCD had very early features of SIRS with mild renal compromise prior to PCD insertion, which subsequently subsided, and all patients recovered completely. The mean hospital stay for these patients is 34.6 days. There were no deaths in this group.

Out of 20 patients, three patients (15\%) required surgical intervention after PCD drainage (Category B). All three referred for PCD with established clinical features of SIRS 


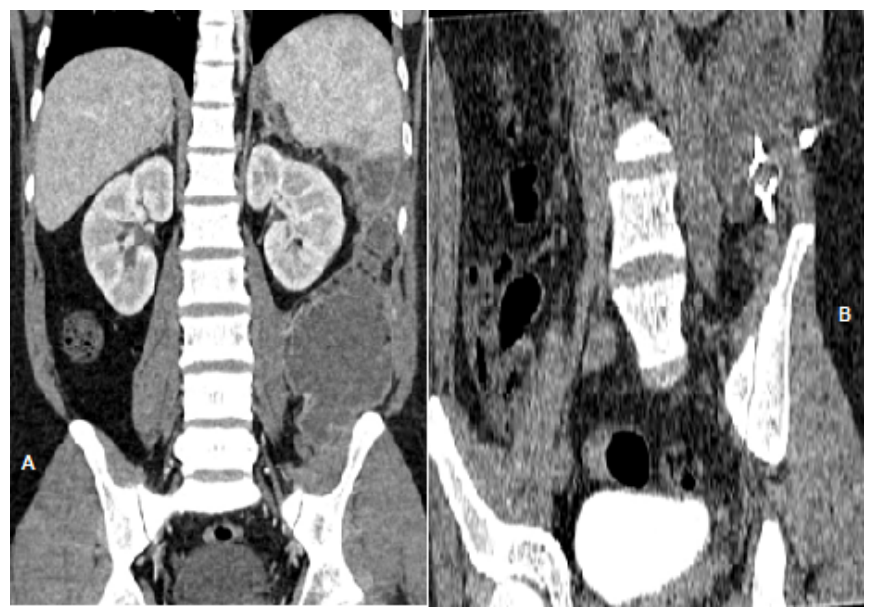

Figure 6: A 43-year-old man with moderately severe necrotizing pancreatitis. A. Coronal contrast-enhanced CT image obtained 13 days after onset of symptoms showing fluid accumulation extending along leftparacolic gutter (represented by the star symbol). B. Coronal plain CT image obtained after insertion of Malecot's catheter into the necrotic collection (depicted with white arrow).

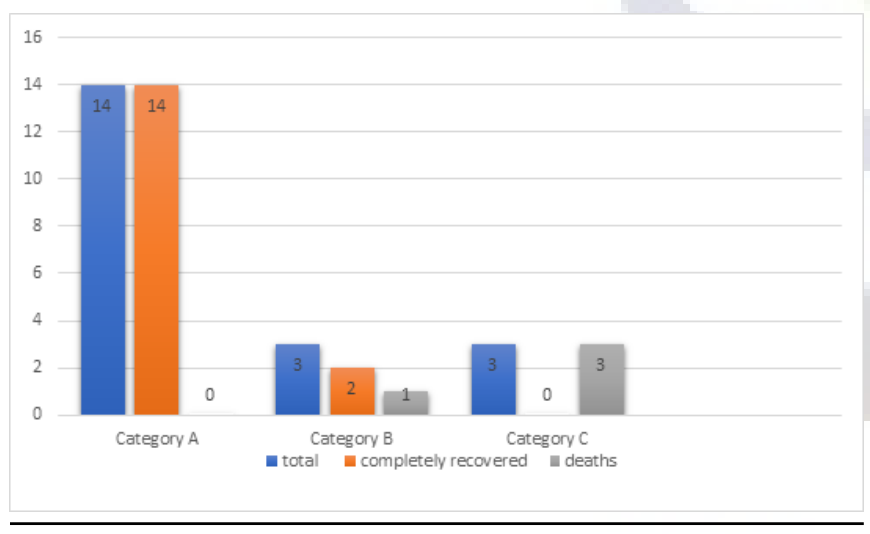

Chart 1: Cases and outcome in different categories

and moderately increased serum creatinine levels. The three patients had undergone open retroperitoneal debridement of necrotic collection, as there was no improvement in their clinical condition, insufficient drainage through drain tube, increasing abdominal pressure, and increasing TLC and creatinine levels. Two out of three were improved post surgically. One patient developed MODS and died in spite of the aggressive medical and surgical management. These patients underwent surgical intervention with a mean of 40 days after hospital admission and a mean of 27 days after the first interventional procedure. The mean hospital stay of the two patients was 84 days.

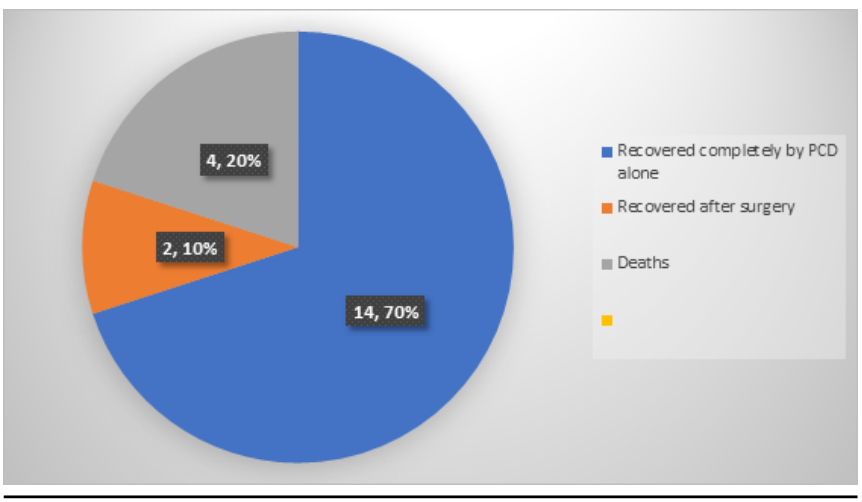

Chart 2: The Clinical outcome in our study

Among 20 patients, 3 patients (15\%) underwent PCD drainage alone because they were not considered as surgical candidates (Category C). All these patients had the severe disease at the time of presentation with features of sepsis and MODS. These patients were intervened with PCD, however, all clinically deteriorated despite the aggressive medical management.

All patients underwent image guided PCD drainage at the earliest possible after admission with a mean duration of 14 days. In our hospital, we intervened at the stage of necrotic collections itself and did not hold back to get collections organized.

\section{Discussion}

Acute pancreatitis is divided into two morphologic subtypes: interstitial edematous pancreatitis and necrotizing pancreatitis. Acute necrotizing pancreatitis is a severe form of acute pancreatitis which is characterized by necrosis within the pancreas and peripancreatic area. It is seen in $20 \%-30 \%$ of patients with acute pancreatitis and historically is associ $\neg$ ated with high rates of morbidity $(34 \%-95 \%)$ and mortality $(2 \%$ $39 \%)$. ${ }^{[1-3]}$

Pathophysiologically, acute pancreatitis is divided into early and late phases. The early phase occurs in the 1 st week after onset, which is manifesting as a systemic inflammatory response. Clinical severity and treatment in this phase are mainly determined on the basis of type and degree of organ failure. The late phase, which generally starts in the 2 nd week and can last for weeks to months, occurs only in patients with moderately severe or severe pancreatitis, as defined by persistent organ failure and by local complications. ${ }^{[4]}$

The revised Atlanta classification system subdivides collections associated with necrotizing pancreatitis as per the time of disease onset. The collection that develops within 4 weeks of onset and lacks a discrete wall is defined as an acute necrotic collection (ANC). A collection that persists after 4 weeks 
and develops a discrete wall is defined as walled-off necrosis (WON). Both an ANC and WON can be sterile or infected. Collections developed in necrotizing pancreatitis consists of fluid and also necrotic tissue of both pancreatic and peripancreatic regions. ${ }^{[4]}$

The diagnosis of infected Acute necrotizing pancreatitis is based on CECT, clinical and laboratory findings. FNA culture and sensitivity has proven positive results in identifying the infections. Intervention is initiated based on imaging findings like gas in collections, clinically persistent fever, and elevated leukocyte count, even though culture results were negative. ${ }^{[8]}$

Few predictive factors, like Ranson, APACHE II scores and CT scores, can be used to evaluate the condition of a patient and help in assessing the clinical success rates or mortality. However, the result of several studies revealed that the CT Severity Index score is not having a good correlation with patient outcome. ${ }^{[9-11]}$

Several studies have proposed that assessing organ failure can be a significant factor in classifying the disease severity and is closely associated with mortality. One study reported that mortality was $0 \%$ in patients with no organ failure, $13 \%$ in patients with single organ failure, and increased significantly to $38 \%$ in patients with MSOF. ${ }^{[12]}$ Another study reported the mortality was $45 \%$ in the patients with MSOF. ${ }^{[13]}$

In Acute pancreatitis, SIRS is the main cause of early complications, whereas fluid collections with subsequent infections are cause for late complications. Clinical criteria for SIRS are 1) Heart rate $>90$ beats/min; 2)Body core temperature $<36^{0} \mathrm{C}$ or $>38^{0} \mathrm{C}$; ; 3) Leucocyte count $<4,000$ or $>12,000 / \mathrm{mm}^{3}$; 4)Respiratory rate $>20 / \mathrm{min}$ or $\mathrm{PCO}_{2}<32 \mathrm{mmHg}$. The presence, extent, and duration of organ failure determine the severity of pancreatitis in the early phase (1st week). The criteria for diagnosis of organ failure are, systolic blood pressure $<90$ $\mathrm{mmHg}, \mathrm{PaO}_{2} \leq 60 \mathrm{mmHg}$, serum creatinine $>2 \mathrm{mg} / \mathrm{dL}$, or gastrointestinal bleeding $>500 \mathrm{~mL} /$ day. ${ }^{[5,16]}$

The current management of acute necrotizing pancreatitis, although remains controversial, the initial treatment strategy has been shifted from aggressive, early surgical debridement to a more conservative and supportive therapy during the initial 2 weeks after the onset of symptoms at most centers. ${ }^{[17]}$

Gotzinger et al. evaluated the cases of 250 patients with acute necrotizing pancreatitis and they found significantly higher mortality among patients operated on within the first 3 weeks after the onset of symptoms than among patients for whom surgery was delayed. ${ }^{[5]}$ Rodriguez et al. confirmed those findings in a similar study with 167 patients, finding that delaying surgery at least 28 days after the onset of symptoms led to a better prognosis. ${ }^{[16]}$ The mortality rate was $5.1 \%$ among a group who underwent surgery more than 28 days after symptom onset and $20.3 \%$ among the group who had been operated on earlier. ${ }^{[16,17]}$
The theoretical basis for PCD is that open surgery might not be tolerated by critically ill patients who are complicated by SIRS and MODS. Image guided PCD can improve their serious clinical condition and postpone surgical intervention. PCD was considered as the first minimally invasive step in the treatment of clinically suspected or diagnosed infective necrotizing pancreatitis with resultant lesser complications. ${ }^{[6,7]}$

Freeny et al. evaluated 34 patients with acute necrotizing pancreatitis in whom the infected pancreatic necrosis was managed with percutaneous catheter drainage the disease resolved without surgery in $47 \%$ of the patients. As important, in $74 \%$ of patients sepsis was controlled, such that the PCD can be considered as a bridge to elective surgery. ${ }^{[18]}$ Wasler et al. proposed in his study that the clinical utility of CT-guided PCD in patients with sterile pancreatic necrosis has no benefit in draining sterile collections versus simple aspiration, however, there might be increased risk of bacterial colonization. However, it is feasible, that a patient with sterile necrosis whose clinical condition continues to worsen in spite of aggressive supportive therapy, might be benefited from percutaneous catheter drainage. ${ }^{[19]}$

In a systematic review study, the size of the drains used varied from 6 to $32 \mathrm{~F}$, and not even a single study showed that large bore drainages are more effective in preventing complications and outcomes.

In one study, among 11 patients with multisystem organ failure, only four (36\%) were treated successfully with CTguided percutaneous catheter drainage alone; five patients (45\%) died. Among 24 patients without multisystem organ failure, 13 (54\%) were treated successfully with CT-guided percutaneous catheter drainage alone; one patient died. ${ }^{[17]}$

In our study, 14 of 20 patients (70\%) with clinically suspected infected pancreatic necrosis were intervened early and treated successfully with CT-guided percutaneous catheter drainage alone. Three out of 20 patients $(15 \%)$ underwent retroperitoneal surgical debridement. Among these three, only one patient died, and two patients recovered. The median time between hospital admission and surgery was 65 days. Initial catheter drainage resulted in avoiding the potentially complicated early surgical intervention, control of sepsis, and decreased mortality, in these patients. Three patients, presented with multi system organ failure who required ICU hospitalisation and ventilation succumbed despite image guided PCD.

In a study, the prevalence of organ failure in acute necrotizing pancreatitis has been reported as 54\% (range, 29-78\%). Patients without organ failure have a $0 \%$ mortality rate; those with single organ failure have median mortality of $3 \%$ (range, $0-8 \%$ ); and those with multisystem organ failure have a median mortality rate of $47 \%$ (range, $28-69 \%$ ). ${ }^{[20]}$ In our study, 4(20\%) patients died; all were complicated by Multi 
system organ failure.

A review study revealed that $38.5 \%$ percent of patients still needed other surgical interventions in spite of PCD. The most common reason was that the necrotic tissue could not be efficiently removed in a seriously ill subset of patients despite the prolonged percutaneous drainage and multiple saline irrigations. The main additional intervention is the surgical approach which includes either open or other minimal- invasive interventional approaches, like percutaneous necrosectomy, videoassisted retroperitoneal debridement (VARD), and retroperitoneal necrosectomy. ${ }^{[6,21-24]}$ In our study, 3 (15\%) patients required retroperitoneal necrosectomy.

\section{Conclusion}

Image guided Percutaneous catheter drainage is a safe and effective technique to drain pancreatic collections. Adequate knowledge regarding the interpretation of imaging findings, appropriate time for intervention, adequate expertise for performing the procedure, close follow up with regular clinical assessment is vital in the management of patients in acute pancreatitis. Early and aggressive intervention helps reduce the morbidity and mortality by preventing the evolution of MODS. However, more extensive studies are required to understand the effect of important confounding prognostic factors in the management and clinical outcome of acute pancreatitis.

\section{References}

1. Baron TH, Morgan DE. Acute Necrotizing Pancreatitis. N Eng J Med. 1999;340(18):1412-1417. Available from: https: //dx.doi.org/10.1056/nejm199905063401807.

2. Beger HG, Rau B, Mayer J, Pralle U. Natural course of acute pancreatitis. World J Surg. 1997;21:130-135. Available from: https://dx.doi.org/10.1007/s002689900204.

3. Shyu JY, Sainani NI, Sahni VA, Chick JF, Chauhan NR, Conwell DL, et al. Necrotizing Pancreatitis: Diagnosis, Imaging, and Intervention. Radiographics. 2014;34(5):1218-1239. Available from: https://dx.doi.org/10.1148/rg.345130012.

4. Foster BR, Jensen KK, Bakis G, Shaaban AM, Coakley FV. Revised Atlanta Classification for Acute Pancreatitis: A Pictorial Essay. Radiographics. 2016;36(3):675-687. Available from: https://dx.doi.org/10.1148/rg.2016150097.

5. Götzinger P, Wamser P, Exner R, Schwanzer E, Jakesz R, Függer R, et al. Surgical Treatment of Severe Acute Pancreatitis: Timing of Operation is Crucial for Survival. Surgical Infections. 2003;4(2):205-211. Available from: https: //dx.doi.org/10.1089/109629603766957004.

6. Ke L, Li J, Hu P, Wang L, Chen H, Zhu Y. Percutaneous catheter drainage in infected pancreatitis necrosis: a systematic review. Indian J Surg. 2016;78(3):221-229. Available from: https://dx.doi.org/10.1007/s12262-016-1495-9.

7. Besselink M, Santvoort HV, Freeman M, Gardner T, Mayerle $\mathrm{J}$, Vege SS, et al. IAP/APA evidence-based guidelines for the management of acute pancreatitis. Pancreatol. 2013;13(4):1-5. Available from: https://doi.org/10.1016/j.pan.2013.07.063.

8. Hollemans RA, van Brunschot S, Bakker OJ, Bollen TL, Timmer R, Besselink MG, et al. Minimally invasive intervention for infected necrosis in acute pancreatitis. Expert Rev Med. 2014;11(6):637-648. Available from: https://dx.doi. org/10.1586/17434440.2014.947271.

9. Tsiotos, Leon LD, Sarr. Long-term outcome of necrotizing pancreatitis treated by necrosectomy. Br J Surg. 1998;85:1650 1653. Available from: https://dx.doi.org/10.1046/j.1365-2168. 1998.00950.x.

10. Freeny PC, Hauptmann E, Althaus SJ, Traverso LW, Sinanan M. Percutaneous CT-guided catheter drainage of infected acute necrotizing pancreatitis: techniques and results. Am J Roentgenol. 1998;170:969-975. Available from: https://dx.doi. org/10.2214/ajr.170.4.9530046.

11. Bruennler T. Outcome of patients with acute, necrotizing pancreatitis requiring drainage-does drainage size matter? World J Gastroenterol. 2008;14(5):725-725. Available from: https://dx.doi.org/10.3748/wjg.14.725.

12. Rocha FG, Benoit E, Zinner MJ, Whang EE, Banks PA, Ashley $\mathrm{SW}$, et al. Impact of radiologic intervention on mortality in necrotizing pancreatitis: the role of organ failure. Arch Surg. 2009;144:261-266. Available from: https://doi.org/10.1001/ archsurg.2008.587.

13. Baudin G, Chassang M, Gelsi E, Novellas S, Bernardin G, Hébuterne X, et al. CT-Guided Percutaneous Catheter Drainage of Acute Infectious Necrotizing Pancreatitis: Assessment of Effectiveness and Safety. Am J Roentgenol. 2012;199(1):192199. Available from: https://dx.doi.org/10.2214/ajr.11.6984.

14. Lorenz J, Bennett S. The Role of Imaging-Guided Percutaneous Procedures in the Multidisciplinary Approach to Treatment of Pancreatic Fluid Collections. Semin Intervent Radiol. 2012;29(04):314-318. Available from: https://dx.doi.org/10. 1055/s-0032-1330066.

15. Sharma P, Sharma S, Yadav A, Rotem E. CT guided percutaneous drainage in necrotizing pancreatitis-highly successful in appropriately selected patients-single center experience. J Pancreas. 2019;20(1):24-33.

16. Rodriguez JR, Razo AO, Targarona J, Thayer SP, Rattner DW, Warshaw AL, et al. Debridement and Closed Packing for Sterile or Infected Necrotizing Pancreatitis. Ann Surg. 2008;247:294-299. Available from: https://dx.doi.org/10. 1097/sla.0b013e31815b6976.

17. Mortelé KJ, Girshman J, Szejnfeld D, Ashley SW, Erturk SM, Banks PA, et al. CT-Guided Percutaneous Catheter Drainage of Acute Necrotizing Pancreatitis: Clinical Experience and Observations in Patients with Sterile and Infected Necrosis. Am J Roentgenol. 2009;192(1):110-116. Available from: https: //dx.doi.org/10.2214/ajr.08.1116.

18. Freeny PC, Hauptmann E, Althaus SJ, Traverso LW, Sinanan M. Percutaneous CT-guided catheter drainage of infected acute necrotizing pancreatitis: techniques and results. Am Jf Roentgenol. 1998;170:969-975. Available from: https://dx.doi. org/10.2214/ajr.170.4.9530046.

19. Walser EM, Nealon WH, Marroquin S, Raza S, Hernandez JA, Vasek J. Sterile Fluid Collections in Acute Pancreatitis: 
Catheter Drainage Versus Simple Aspiration. CardioVas Interv Radiol. 2006;29:102-107. Available from: https://dx.doi.org/ 10.1007/s00270-004-0220-4.

20. Banks PA, Freeman ML. Practice Parameters Committee of the American College of Gastroenterology. Practice guidelines in acute pancreatitis. Am J gastroenterol. 2006;101(10):2379 400.

21. Tong Z, Liw, Yuw, Gengy, Kel N. Percutaneous catheter drainage for infective pancreatic necrosis: is it always the first choice for all patients? Pancreas. 2012;41(2):302-305.

22. Tong ZH, Li WQ, Yu WK, Wang XY, Ye XH, Nie Y. The clinical effectiveness of percutaneous drainage and laparotomy for patients with infective pancreatic necrosis. Zhonghua Wai Ke Za Zhi. 2010;48(18):1387-1391.

23. Porter KK, Zaheer A, Kamel IR, Horowitz JM, Arif-Tiwari H, Bartel TB, et al. ACR Appropriateness Criteria ${ }^{\circledR}$ Acute Pancreatitis. J Am Coll Radiol. 2019;16(11):S316-S330. Available from: https://dx.doi.org/10.1016/j.jacr.2019.05.017.

24. Türkvatan A, Erden A, Türkoğlu MA, Seçil M, Yener Ö. Imaging of acute pancreatitis and its complications. Part 1:
Acute pancreatitis. Diag Interv Imag. 2015;96(2):151-160. Available from: https://dx.doi.org/10.1016/j.diii.2013.12.017.

Copyright: (C) the author(s), 2020. It is an open-access article distributed under the terms of the Creative Commons Attribution License (CC BY 4.0), which permits authors to retain ownership of the copyright for their content, and allow anyone to download, reuse, reprint, modify, distribute and/or copy the content as long as the original authors and source are cited.

How to cite this article: Sudha CR, Samireddypalle Y, Reddy UV, Dembla S, Varty GP, Dhawan AS, Vikrama AKS. Role of Early Intervention in Improving the Clinical Outcome of Acute Pancreatitis by Image Guided Percutaneous Catheter Drainage of Pancreatic Collections. Asian J. Med. Radiol. Res. 2020;8(1): $132-138$.

DOI: dx.doi.org/10.47009/ajmrr.2020.8.1.24

Source of Support: Nil, Conflict of Interest: None declared. 